\title{
Captured Macro-embolus of Fractured Atheromatous Plaque by the Embolic Protection Device during Carotid Stent Assisted Angioplasty
}

\author{
Mun Chul Kim, MD², Shelby Bennett, MD', Richard Farb, MD, \\ Sydney Croul, MD, Seon-Kyu Lee, MD'
}

The authors present a case in which macro-embolus from the ruptured atheromatous plaque developed during carotid artery stenting (CAS). A 63-year-old man who had suffered a left middle cerebral artery territory infarction had significant proximal left internal carotid artery stenosis required CAS procedure. Immediate after stent deployment, the patient showed abrupt neurological deterioration with $12 \times 3 \mathrm{~mm}$ sized macro-embolus which was caught by the embolus protection device (EPD). Retrieval of the macro-embolus was performed safely and the patient recovered to pre-procedure status. Macroembolus can be resulted during the CAS. The EPD can capture the macro-embolus and safe removal is technically feasible.

Key Words : Carotid stenting; Embolus protection device; Atheromatous plaque

One of the most important risks of carotid artery stenting (CAS) is periprocedural embolic stroke which is believed to be related to the distal embolization of fragmented debris of plaque. The fear of the distal embolization during CAS has generated the development of the embolus protection device (EPD). The

\footnotetext{
'Department of Radiology, The University of Chicago, Chicago, IL, USA

${ }^{2}$ Department of Neurosurgery, Pohang Stroke and Spine Hospital, Pohang, Korea

${ }^{3}$ Department of Medical Imaging and ${ }^{4}$ Pathology, Toronto Western Hospital, Toronto, ON, Canada

Received December 27, 2012; accepted after revision February 7, 2013.

Correspondence to: Seon-Kyu Lee, MD, PhD, Neurointerventional Service, Department of Radiology, 5841 S.Maryland Ave. MC 2026, Chicago, IL, 60637, USA.

Tel. +1.773.702.3654 Fax. +1.773.702.1161

E-mail: sklee@uchicago.edu

This is an Open Access article distributed under the terms of the Creative Commons Attribution Non-Commercial License (http://creativecommons.org/licenses/by-nc/3.0) which permits unrestricted non-commercial use, distribution, and reproduction in any medium, provided the original work is properly cited.
}

reported sizes of captured emboli were up to $5.2 \mathrm{~mm}$ $[1,2]$, but as far as authors know, there is no report of captured macro-embolus which is larger than $1 \mathrm{~cm}$ in length. We report a case with the successful use of the EPD, which captured the macro-embolus during the CAS for symptomatic carotid artery stenosis.

\section{CASE REPORT}

A 63-year-old man who had suffered the left middle cerebral artery (MCA) territory ischemic stroke made a good clinical recovery. Pre-procedure neurologic examination showed minimal right upper extremity motor weakness with mild speech and cognitive dysfunction. The Magnetic Resonance Imaging and MR angiography showed about $90 \%$ luminal stenosis on the left proximal internal carotid artery (ICA). The patient agreed to be enrolled into the Carotid Revascularization Endarterectomy and Stent Trial (CREST) and he was randomized to Stenting arm. The CAS was performed under the CREST Stenting protocol by a CREST certified interventionist under 


\section{Embolus During Carotid Artery Stenting}

neuroleptic anesthesia.

Diagnostic angiography showed approximately $70 \%$ of luminal stenosis by NASCET criteria (Fig. 1). A 6 Fr Shuttle ${ }^{\circledR}$ Guiding catheter (Boston Scientific, USA) was placed at the proximal left common carotid artery. Subsequently a $4.5 \mathrm{~mm}$ sized Accunet ${ }^{\circledR}$ protection device (Guidant Co. USA) was introduced thru the guiding catheter and placed at the distal cervical segment of the left ICA. A $4 \times 15 \mathrm{~mm}$ sized Voyager angioplasty balloon (Abbott Vascular, USA) was used to perform pre-stent angioplasty and the balloon was inflated upto $8 \mathrm{~atm}$. The post-angioplasty angiography

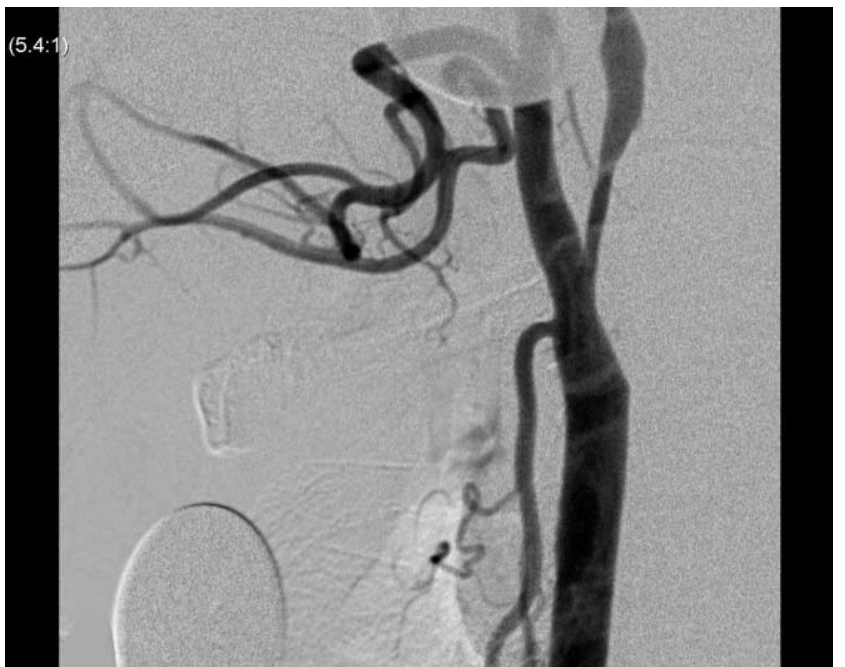

Fig. 1. Lateral view of the left CCA angiogram shows about $70 \%$ of smooth luminal stenosis without angiographic evidence plaque ulcer or irregularity. which showed dissection of atheromatous plaque with contrast filling (Fig. 2). A $7 \times 40 \mathrm{~mm}$ sized RXAcculink ${ }^{\circledR}$ Carotid stent (Guidant Co. USA) was then introduced by coaxial manner and deployed across the stenotic segment. Immediate after the stent deployment, the patient's showed acute expressive dysphasia, disorientation and decreased verbal comprehension.

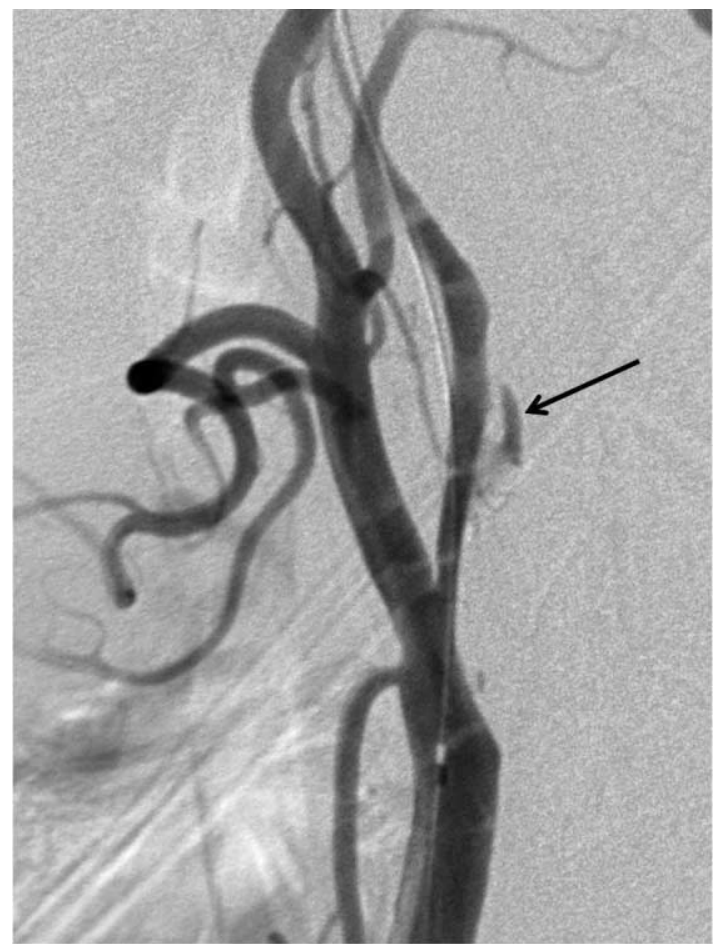

Fig. 2. Lateral view of post-angioplasty CCA angiogram shows development of dissection on the atheromatous plaque (arrow).
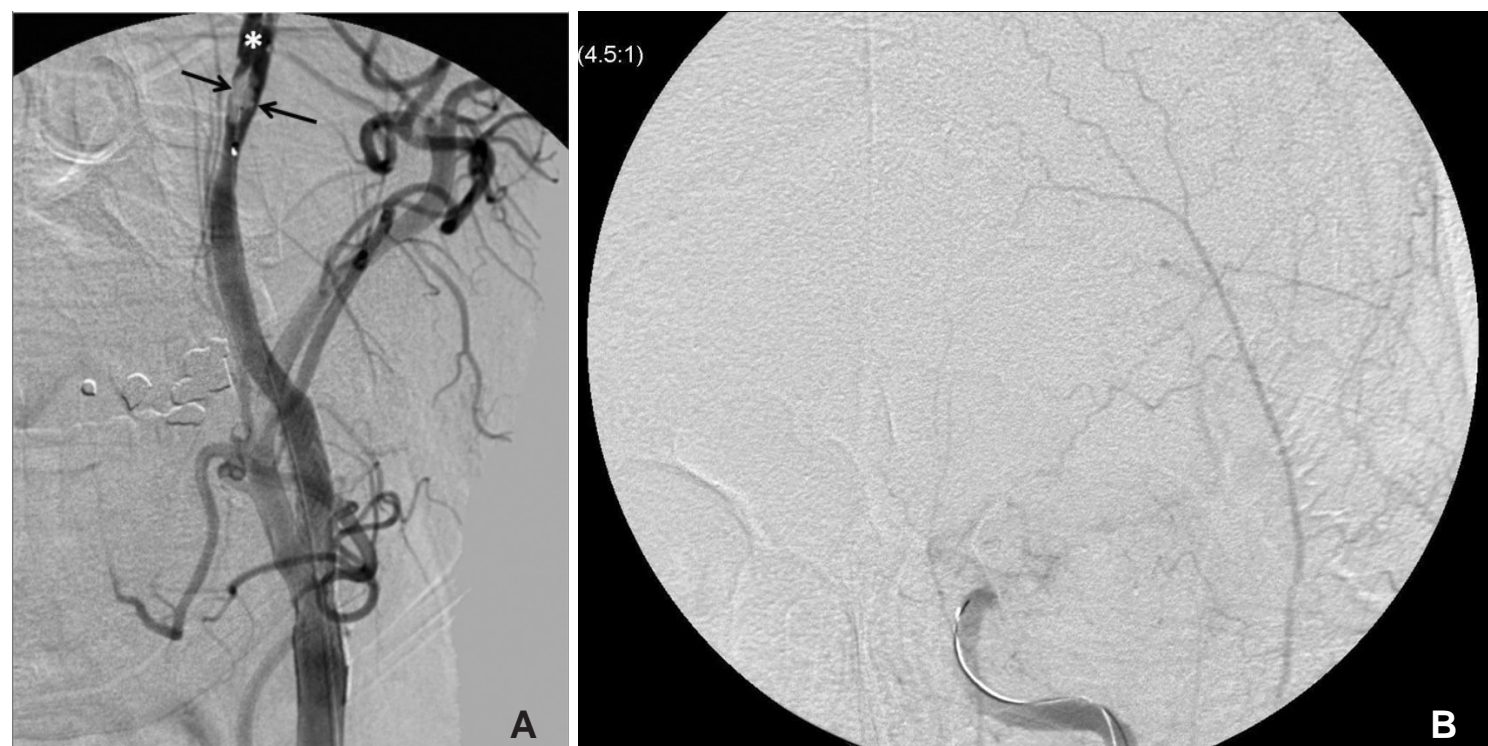

Fig. 3. After stent deployment, the left CCA angiogram AP view $(\mathbf{A})$ shows significant very dense contrast accumulation $\left({ }^{*}\right)$ beyond the spindle shaped filling defect (arrows) captured in the EPD indicating significant slow flow beyond the filling defect. Subsequent intracranial AP view (B) showed slow filling of distal left ICA. 
Mun Chul Kim, et al.

He also developed right lower extremity weakness. Immediate post stenting angiography of the left ICA showed about $4 \times 10 \mathrm{~mm}$ sized spindle shaped filling

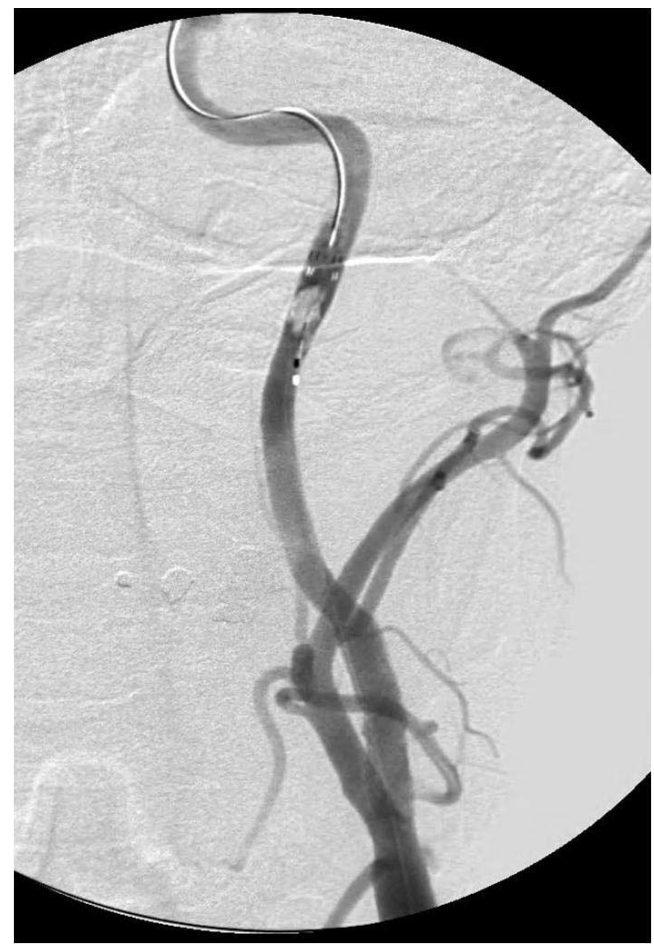

Fig. 4. 10 minutes after Reopro ${ }^{\circledR}$ infusion, the forward flow through the filling defect shows re-established on the left CCA AP view.

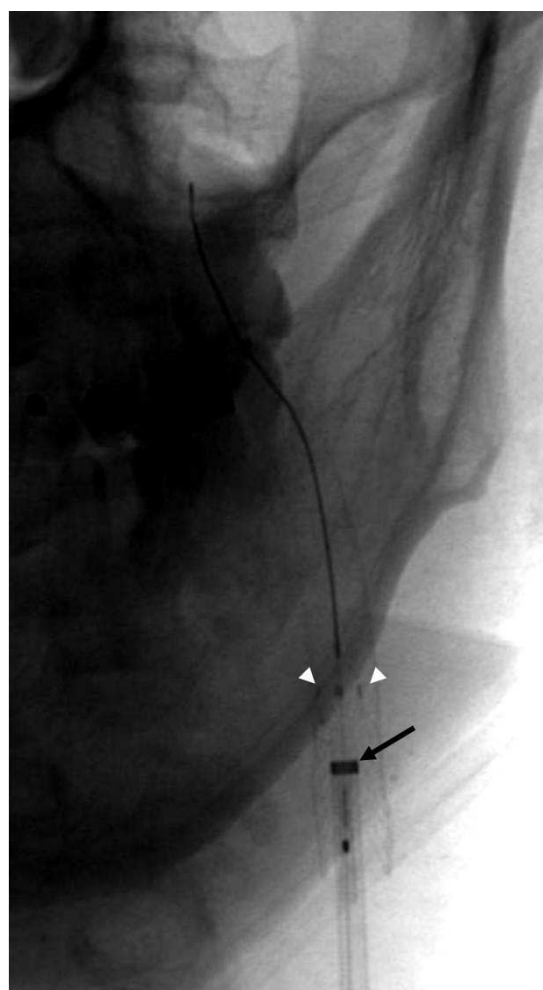

Fig. 5. AP spot film during the removal of the Rx-Accunet ${ }^{\circledR}, R x-$ Accunet Retrieval device $2{ }^{\circledR}$ and Shuttle ${ }^{\circledR}$ sheath. Note the 4 radio-opaque markers of the $\mathrm{Rx}$-Accunet ${ }^{\circledR}$ are not completely opposed (white arrow heads) and the position of the Shuttle ${ }^{\mathbb{R}}$ sheath (arrow) which was advanced until the Rx-Accunet ${ }^{\circledR}$ wires can be straight enough not to be caught by the deployed stent struts.
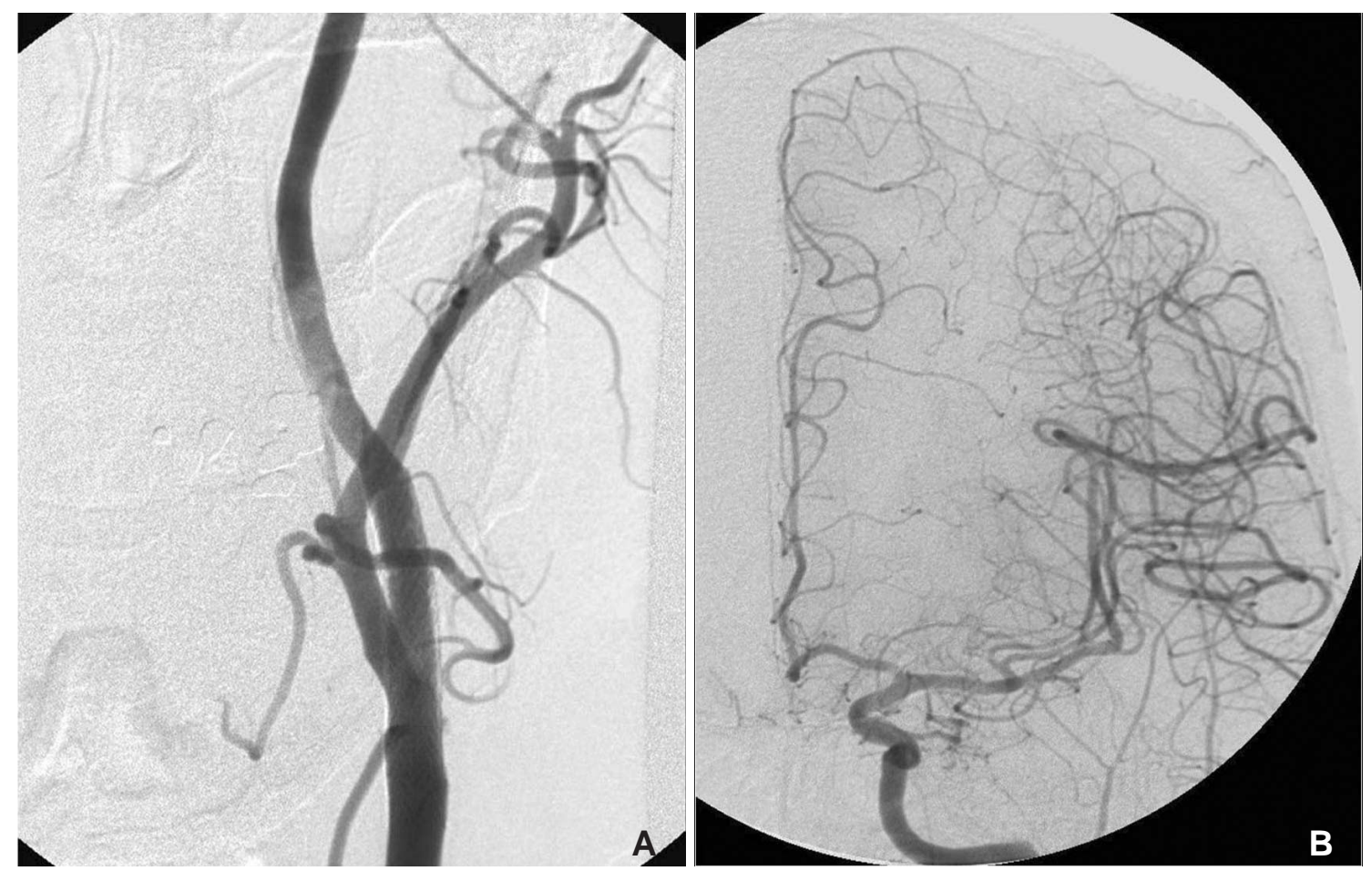

Fig. 6. After the removal of the filling defect, AP view of extracranial (A) and intracranial (B) circulation on the left CCA angiogram show no additional filling defect. 


\section{Embolus During Carotid Artery Stenting}

defect (Fig. 3A), which was captured by the protection device. The intracranial circulation showed significantly decreased blood flow (Fig. 3B). Assuming the lesion could be a mixture of multiple fragmented plaque debris associated with platelet aggregation, we started Reopro ${ }^{\circledR}$ (Lilly, USA) infusion $(0.25 \mathrm{ug} / \mathrm{kg}$ bolus IV) to prevent further potential progression of platelet aggregation. After 10 minutes of Reopro ${ }^{\circledR}$ infusion, the patient's intracranial flow was reestablished. However, even after 25 minutes of Reopro infusion, the size of filling defect did not show interval change (Fig. 4). Thus, we thought the filling defect would more likely to be an organized embolus from the plaque than platelet aggregation, we decided removing the lesion with the protection device to prevent fracturing the lesion during the retrieval process. To facilitate the removal of the filling defect, we angioplastied the stented left ICA with $6 \times 20 \mathrm{~mm}$ sized balloon catheter. Then, we carefully advanced Rx-Accunet retrieval device $2^{\circledR}$ (Guidant, USA) and tried to pull the EPD and retrieval device down thru the stent without squeezing the filling defect. However, since the retrieval device could not completely capture the EPD, a couple of the EPD wires were always catching stent struts during the removal process. Thus, we used the 6 Fr Shuttle ${ }^{\circledR}$ guiding catheter as a supporting retrieval device. We carefully advanced the 6 Fr Shuttle ${ }^{\circledR}$ catheter up to the proximal $1 / 3$ of the retrieval device (Fig. 5) without squeezing the filling defect and successfully pulled the whole system through the stent toward the descending aorta. At this point, we removed protection device and retrieval device thru the guiding catheter but left shuttle at the descending thoracic aorta. Post-embolus removal angiography showed no evidence of residual emboli on both extracranial and intracranial circulations (Fig. 6). The retrieved embolus was $12 \times 3 \mathrm{~mm}$ in size (Fig. 7A). The histopathologic examination showed relative acellular matrix with frequent cholesterol clefts, focal calcifications and focal fibrous strands which are consistent with typical pathologic findings of organized atherosclerotic plaque (Fig. 7B and $\mathrm{C}$ ). The patient was discharged from the hospital post-CAS 2 nd day without additional neurologic deficit to his pre-procedure deficits. Post-CAS 30th day clinical follow up showed improved speech and cognitive dysfunction than pre-CAS status.

\section{DISCUSSION}

Many studies have shown the histopathologic analysis of the retrieved embolic particles. Gray et al. [3] have reported that EPDs captured atherosclerotic debris in $57 \%$ of samples and debris consisted of foam
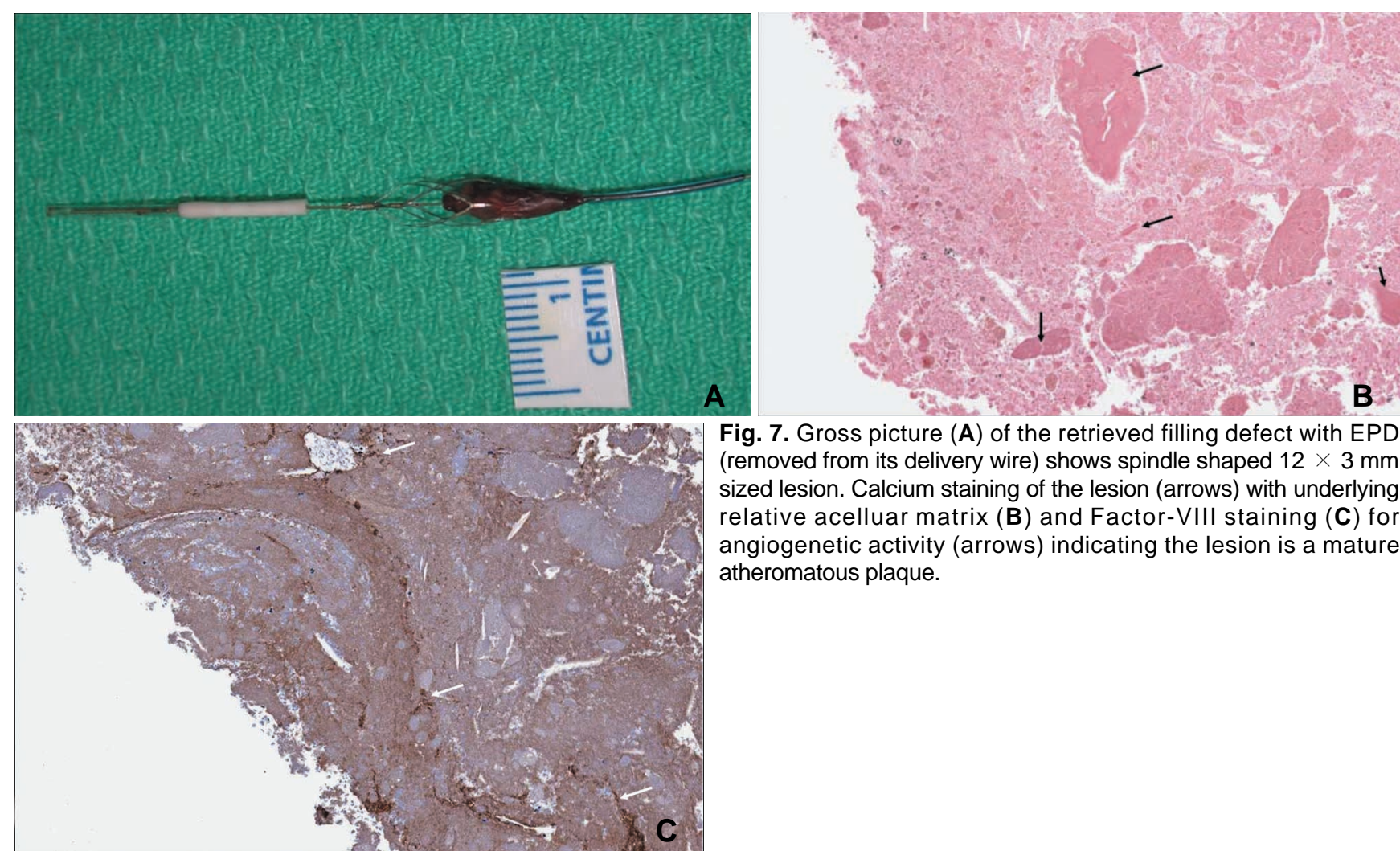

Fig. 7. Gross picture $(\mathbf{A})$ of the retrieved filling defect with EPD (removed from its delivery wire) shows spindle shaped $12 \times 3 \mathrm{~mm}$ sized lesion. Calcium staining of the lesion (arrows) with underlying relative acelluar matrix (B) and Factor-VIII staining (C) for angiogenetic activity (arrows) indicating the lesion is a mature atheromatous plaque. 


\section{Mun Chul Kim, et al.}

cells, smooth muscle cells, cholesterol, collagen/elastin, and platelet/fibrin. Angelini et al. [1] have reported that particles could be detected in $83.7 \%$ microscopically, and consisted of soft acellular and amorphous material characterized by lipid-rich macrophages and cholesterol clefts. Hill et al. [4] have demonstrated the similar histopathologic results. In our case, the embolus was relatively acellular with frequent cholesterol clefts, focal calcification, and focal fibrous strands which are typical histopathologic features of mature atheromatous plaque.

The size of retrieved particles has been reported up to $5.2 \mathrm{~mm}$ in length $[1,2,4]$. As far as authors know, 12 $\times 3 \mathrm{~mm}$ particle size is the largest particle in the published reports. The development of such large embolic particle could be the result of the athermanous plaque squeezing and rupture. Lipid-rich and inflamed plaques are vulnerable to rupture. It could be exposed to the blood stream by squeezing during pre-stent balloon angioplasty, which made subintimal dissection and followed by rupture to the distal circulation during the CAS. Since the stent starts deploying distal to proximal direction, the macroembolus in our case could more likely be occurred either during the pre-stent angioplasty or during the advancement of stent rather than during the stent deployment. However, it could also be possible that the macro-embolus was squeezed during the stent deployment from distal to proximal and the plaque took off immediate after complete deployment of the stent through the proximal end of the stent toward distal through the deployed stent.

Removal of macro-embolus with EPD and the retrieval catheter through the deployed stent was possible using the $6 \mathrm{Fr}$ Shuttle ${ }^{\circledR}$ guiding catheter as a supporting device. Since it has inner diameter of 0.087 inch $(2.2 \mathrm{~mm})$, it was possible to safely capture the EPD without squeezing the macroembolus and successfully avoid potential catchment of EPD wires to the deployed stent's struts.

Our usage of Reopro ${ }^{\circledR}$ before the retrieval of the EPD which captured the macroembolus could be debatable. Instead of using Reopro ${ }^{\circledR}$, the filling defect could have been retrieved with EPD immediately considering the patient was symptomatic. We thought the most of filling defects were mixture of fragmented debris and acute platelet aggregation, thus we would like to clarify the real size of fragmented debris before we attempted to remove the filling defect. For example, if the size of the filling defect gets smaller after resolving potential platelet aggregation component we may be able to remove the EPD only. In retrospect, since the filling defect was mainly a macroembolus without significant platelet aggregation, immediate removal of the EPD could have relieved the occlusion effect of left ICA by the macroembolus.

The main purpose of carotid revascularization such as CAS and carotid endarterectomy (CEA) is to reduce the risk of future stroke, and the efficacy of these procedures is mainly depends on the peri-procedural complication risks especially periprocedural ischemic stroke. Although a multicenter prospective randomized trial [SPACE (Stent-Supported Percutaneous Angioplasty of the Carotid Artery versus Endarterectomy) trial] did not support the need for using EPD [5], there are many reports which demonstrated clinical benefits of using EPD in CAS. The Carotid and Vertebral Artery Trnasluminal Angioplasty Study (CAVATAS) reported similar postoperative 30 days stroke rates of CAS and CEA as $10 \%$ and $9.9 \%$ respectively, without protection devices [6]. The SAPPHIRE (Stenting and Angioplasty with Protection in Patients at High Risk for Endarterectomy) study found that patients who underwent CAS using an EPD had significantly better results with respect to the primary endpoint of composite death, stroke and MI rate than those of CEA. The cumulative major adverse event incidence of the CAS and CEA of the SAPPHIRE trial were $12 \%$ and $20.1 \%$ respectively [7]. Global CAS registry analysis showed that CAS with EPDs yields a significant lower adverse event rate than CAS without protection (2.2\% vs. $5.3 \%$ rate of strokes and procedure related deaths) [8]. Kastrup et al. [9], who systematically reviewed single-center CAS studies, revealed the combined stroke and death rate within 30 days in both symptomatic and asymptomatic patients was $1.8 \%$ in patients treated with cerebral protection devices compared with $5.5 \%$ in patients treated without cerebral protection devices. They concluded that protection devices appear to reduce thromboembolic complication during CAS. In addition to these reports, our case report of capturing a large embolic particle with EPD may provide additional support on the potential benefits of using the EPD during the CAS procedure.

In conclusion, we demonstrated the possibility of developing macro-embolus from atheromatous plaque disruption during the CAS procedure. The usage of the EPD during the CAS procedure prevented potential complications including significant cerebral infarction from a macro-embolus. In addition, it is technically feasible to safely retrieve the macro-embolus using guiding catheter as a supporting device for EPD 


\section{Embolus During Carotid Artery Stenting}

\section{removal.}

\section{References}

1. Angelini A, Reimers B, Barbera MD, Sacca S, Pasquetto G, Cernetti $\mathrm{C}$, et al. Cerebral protection during carotid artery stenting: collection and histopathologic analysis of embolized debris. Stroke 2002;33:456-461

2. Whitlow PL, Lylyk P, Londero H, Mendiz OA, Mathias K, Jaeger $\mathrm{H}$, et al. Carotid artery stenting protected with an emboli containment system. Stroke 2002;33:1308-1314

3. Gray WA, Hopkins LN, Yadav S, Davis T, Wholey M, Atkinson $\mathrm{R}$, et al. for the ARCHeR Trial Collaborators. Protected carotid stenting in high-surgical-risk patients: the ARCHeR results. $J$ Vasc Surg 2006;44:258-69

4. Hill MD, Morrish W, Soulez G, Nevelsteen A, Maleux G, Rogers $\mathrm{C}$, et al. for the MAVErIC International Investigators. Multicenter evaluation of a self-expanding carotid stent system with distal protection in the treatment of carotid stenosis. AJNR Am J Neuroradiol 2006;27:759-765

5. Jansen O, Fiehler J, Hartmann M, Bruckmann H. Protection or nonprotection in carotid stent angioplasty: the influence of interventional techniques on outcome data from the SPACE trial. Stroke 2009;40:841-846

6. Brown M, Rogers J, Bland J. Endovascular versus surgical treatment in patients with carotid stenosis in the Carotid and Vertebral Artery Transluminal Angioplasty Study (CAVATAS): a randomized trial. Lancet 2001;357:1729-1737

7. Yadav J, Wholey M, Kuntz R, Fayad P, Katzen B, Mishkel G, et al. For the stenting and angioplasty with protection in patients at high risk for endarterectomy investigator. Protected carotid-artery stenting versus endarterectomy in high-risk patients. $N$ Engl J Med 2004;351:1493-1501

8. Wholey Michael H, Al-Mubarak N, Wholey Mark H. Updated review of the global carotid artery stent registry. Catheter Cardiovasc Interv 2003;60:259-266

9. Kastrup A, Groschel K, Krapf H, Brehm BR, Dichgans J, Schulz JB. Early outcome of carotid angioplasty and stenting with and without cerebral protection devices: a systematic review of the literature. Stroke 2003;34:813-819 